\title{
IoT-based Water Quality Monitoring
}

\author{
Wen-Tsai Sung, ${ }^{1}$ Fathria Nurul Fadillah, ${ }^{1}$ and Sung-Jung Hsiao ${ }^{2 *}$ \\ ${ }^{1}$ Department of Electrical Engineering, National Chin-Yi University of Technology, \\ No. 57, Sec. 2, Zhongshan Rd., Taiping Dist., Taichung 411030, Taiwan \\ ${ }^{2}$ Department of Information Technology, Takming University of Science and Technology, \\ No. 56, Sec. 1, Huanshan Rd., Neihu District, Taipei City 11451, Taiwan
}

(Received February 24, 2021; accepted June 18, 2021)

Keywords: IoT, water quality, physiochemical sensors, real time, Arduino

Water pollution has become a major issue over the last few years. Constant water monitoring will be important in dealing with this issue since water is essential to human beings. The purpose of this research is to develop an effective IoT system for monitoring the quality of water in real time using physiochemical sensors. The system is implemented using Arduino as the microcontroller and turbidity, temperature, $\mathrm{pH}$, conductivity, and total dissolved solid (TDS) sensors. The information from each sensor is sent over the internet. Experiments are performed on water quality data acquisition, online data transfer, monitoring, recording, and analysis. The results of the experiments show that the water sources in this study satisfy the parameters of good water quality. In addition, our team proposed a water quality model for running water based on diversion-method-related experiments.

\section{Introduction}

The internet has become essential to the world community, allowing people to communicate with each other very easily and quickly. The internet is not just for connecting people, and objects ("things") can also communicate with other objects. This concept is called the Internet of Things (IoT), where objects have the ability to transfer data over the internet without requiring human-to-human or human-to-computer interaction. The IoT is usually supported by a device or controller that is used to control sensors and actuators, such as Intel Edison, Intel Galileo, Raspberry Pi, or an Arduino-based processor. By using these boards, data from sensors can be monitored and controlled remotely via the internet. ${ }^{(1)}$

Drinking water is an essential requirement for all human beings, and more than $60 \%$ of the human body is made up of water. Utilities providing drinking water face new challenges in realtime operation because of issues such as limited water resources, a growing population, and aging infrastructure. ${ }^{(2)}$ Seventy percent of the surface of the earth is made up of water, which is used for drinking, swimming, and fishing, and by industry and agriculture.

It is difficult to measure water quality. The water on the earth consists of a huge network of rivers, springs, wells, swamps, lakes, wetlands, estuaries, and bays, and the degree of pollution

*Corresponding author: e-mail: sungjung@gs.takming.edu.tw https://doi.org/10.18494/SAM.2021.3342 
varies between different bodies of water. Water quality measurements are necessary to evaluate the physical, chemical, biological, and microbiological characteristics of water. Keeping track of water quality is a major challenge because large amounts of chemicals used in day-to-day activities and in industry ultimately make their way into water. At present, there are few pure water resources on the earth, and many bodies of water are polluted. Water pollution greatly affects the health and survival of humans. With increasing globalization, the world is facing the problems of water demand and contamination.

In Palembang, South Sumatra, Indonesia, water tanks are still used, and water may become cloudy owing to the lack of tank maintenance and cleaning. In addition, there is no system to prevent water cloudiness or to provide advance notification to residents of water problems. This inspired us to use the concept of the IoT to provide instant notifications to residents. The system proposed in this paper enables the measurement of turbidity, temperature, $\mathrm{pH}$, conductivity, and total dissolved solid (TDS) of water to establish whether it is suitable for normal use. The sensors used are turbidity, temperature, $\mathrm{pH}$, conductivity, and TDS sensors. The information from each sensor is sent over the internet. Experiments are performed on water quality data acquisition, online data transfer, monitoring, recording, and analysis. The system is currently in use in the Engineering Building of National Chin-Yi University of Technology, Taichung, Taiwan.

\section{Related Work}

Sachio et al. proposed an IoT system for controlling the water level in water containers using an ultrasonic sensor. ${ }^{(1)}$ They experimentally evaluated the accuracy of ultrasonic sensors in reading the water level and found that the accuracy of the sensors increased when there was a longer delay and the surface of the measured water was flat.

Vijayakumar and Ramya proposed the real-time monitoring of water quality using temperature, $\mathrm{pH}$, turbidity, conductivity, and dissolved oxygen sensors. ${ }^{(2)}$ They used Raspberry $\mathrm{Pi}$ as the controller. They conducted experiments on monitoring water quality on the internet by using cloud computing. The results obtained with this system could be viewed using a separate IP address, making it suitable for environment and ecosystem monitoring, for example.

Jadhav et al. proposed a monitoring system of water usage in industry using the IoT. ${ }^{(3)}$ They constructed an automated water monitoring wireless system to track purchased water. As a result, they were able to monitor the water level and water flow continuously and from anywhere using a mobile phone that allowed users to access real-time data.

Al-Khashab et al. proposed a monitoring system of drinking water in Mosul City through the IoT using physiochemical sensors and Arduino UNO as the microcontroller. ${ }^{(4)}$ They compared the results obtained using the proposed system and in the laboratory and found this system to be very efficient and useful in practice as the water could be tested instantly.

Redwan et al. proposed an exploratory approach to monitor the quality of a water supply through IoT technology and user-friendly systems to reduce the wastage of water. ${ }^{(5)}$ They used photovoltaic panels to power the microcontroller and a liquid crystal display (LCD) to show the output of the system, the data of which were sent to the cloud. A water pump was used to control the supply of water. 
Taru and Karwankar proposed a water monitoring system using Arduino and LabVIEW to remotely monitor and control water quality using three sensors: a $\mathrm{pH}$ sensor, a temperature sensor, and a turbidity sensor. ${ }^{(6)}$ They found that the system was highly economical and reliable for water monitoring as well as easy to operate and install, and also flexible and expandable to industrial use.

\section{Proposed System}

\subsection{System architecture}

Figure 1 shows the system architecture used in this study. In the physical layer, sensors are connected to the network layer comprising Arduino UNO (the microcontroller) and its Wi-Fi module. The sensors monitor the environment and gather sensing data. These data are sent to the cloud for safe storage. All devices can be connected to each other and to the internet through the Wi-Fi module. In the network layer, there is also a microcontroller that makes all the devices work together as a system. This microcontroller consists of hardware and software. The sensing data are sent to the Arduino UNO microcontroller to be processed. All the sensor readings are shown on ThingSpeak, which users can access from their personal computer or mobile phone. ${ }^{(7,8)}$

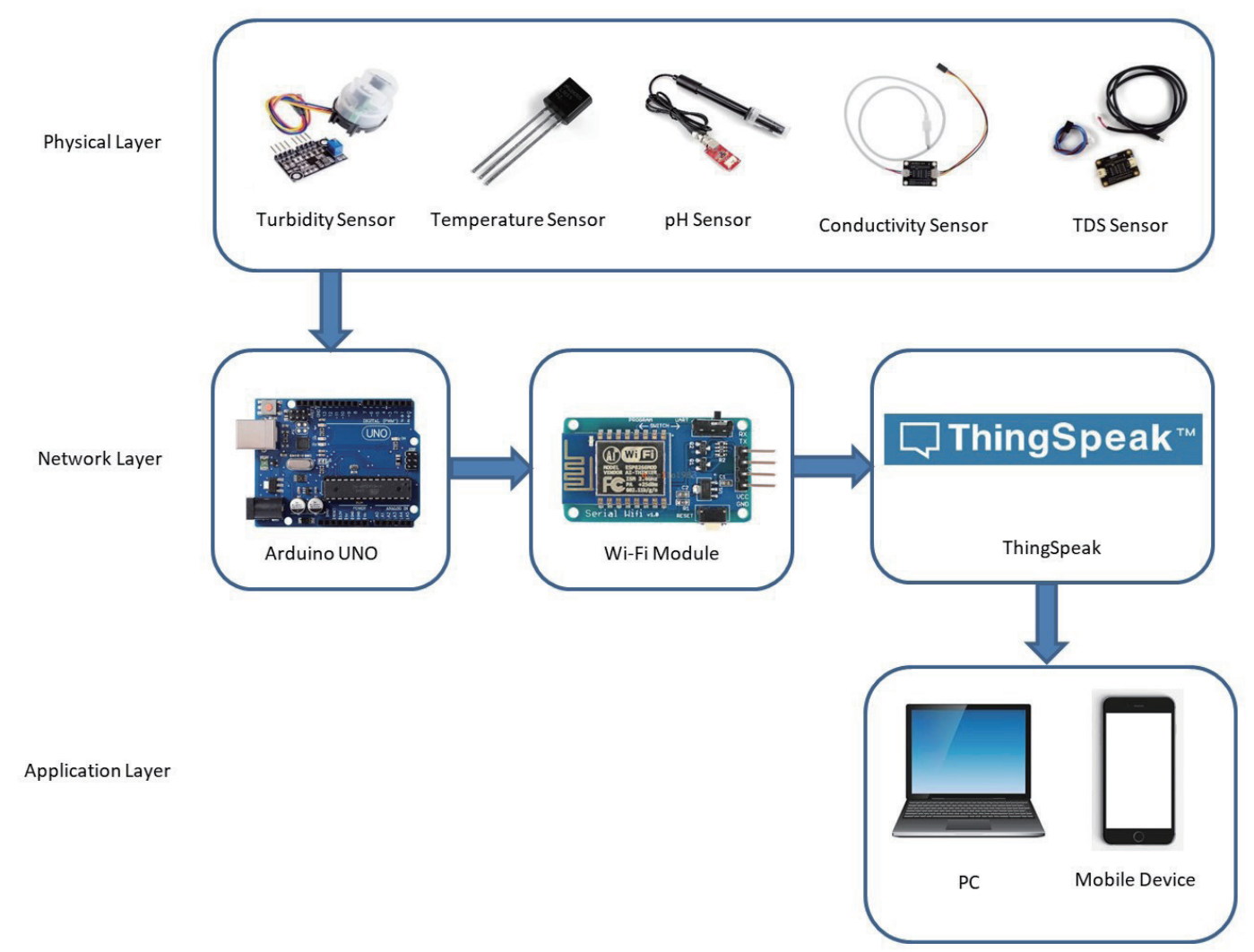

Fig. 1. (Color online) System architecture. 


\subsection{Hardware}

\subsubsection{Arduino UNO}

Figure 2 shows an Arduino UNO board. Arduino is an open source development platform that is relatively simple and easy to use with high-level language that is similar to Java and C language.

\subsubsection{Wi-Fi module}

The ESP 8266 Wi-Fi module (Fig. 3) permits microcontrollers to access a Wi-Fi arrangement and provides an independent and total Wi-Fi organizing arrangement. ${ }^{(9,10)}$

\subsubsection{Temperature sensor}

Figure 4 shows an LM35 temperature sensor, which is a typical temperature sensor and is used to measure the temperature of water in this study. The output voltage can easily be converted to a temperature reading in degrees Celsius. This sensor can measure temperatures from -55 to $150{ }^{\circ} \mathrm{C}$, and its accuracy is very high when operated at optimal temperature and humidity levels..$^{(11,12)}$

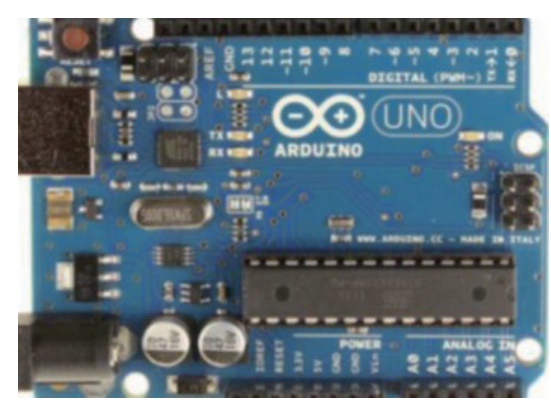

Fig. 2. (Color online) Arduino UNO board.

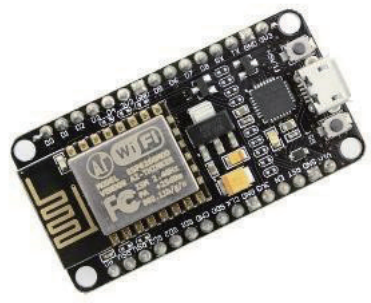

Fig. 3. (Color online) ESP 8266 Wi-Fi module.

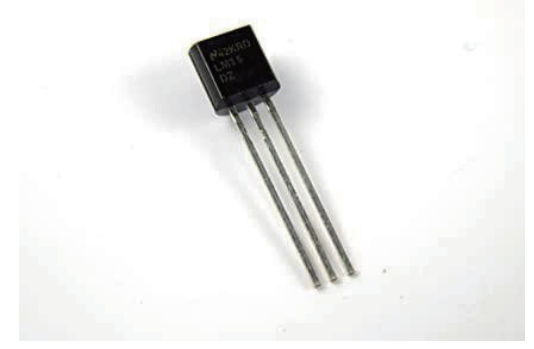

Fig. 4. (Color online) LM35 temperature sensor. 


\subsection{4 pH sensor}

An SKU:SEN0161 pH sensor is used in this study to determine the hydrogen ion concentration in water to determine its alkalinity or acidity, i.e., $\mathrm{pH}$ value. The $\mathrm{pH}$ value for a liquid is between 0 to 14 . The $\mathrm{pH}$ sensor has three pins for the power, ground, and output. Figure 5 presents the sensor and its connection.

\subsubsection{TDS sensor}

TDS indicates the total amount of minerals, salts, and metals dissolved in water. The TDS sensor used in this study has two separate electrodes that are inserted into the water to measure the electrical charge (EC), and the result is converted into concentration in ppm. This is possible because all dissolved solids have an EC that can flow between the electrodes. In the case of pure water containing no soluble materials, the sensor will not conduct EC and it will show a reading of 0 ppm. Figure 6 shows a common SEN0244 board of a TDS sensor.

\subsubsection{Conductivity sensor}

Conductivity indicates the ionic strength of a chemical solution and is directly associated with salinity. Figure 7 shows a common conductivity sensor. This sensor monitors the amount of nutrients, salts, or impurities in the water. The conductivity increases with the number of dissolved ions in the water. ${ }^{(13,14)}$

\subsubsection{Turbidity sensor}

The probe on the turbidity sensor used in the proposed system works by sending a light beam into the water, and the amount of light reflected is used to determine the particle density in the water. The more light that is reflected, the more particles are present in the water. Figure 8 shows the turbidity sensor.

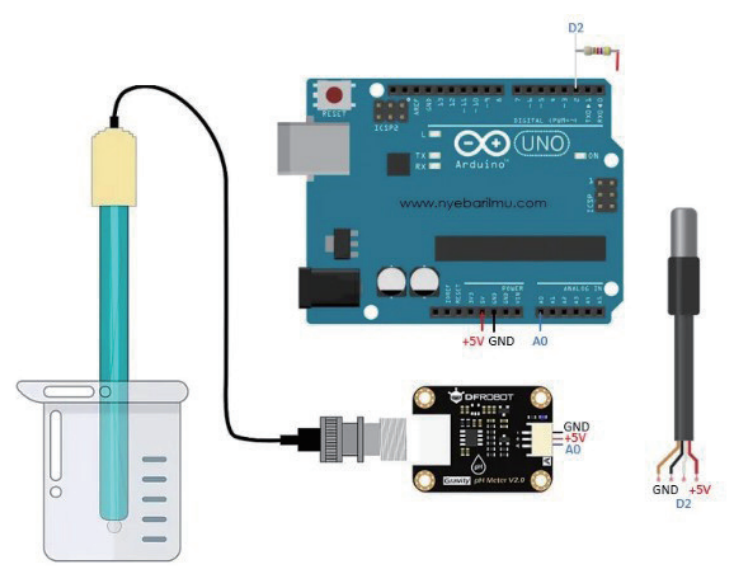

Fig. 5. (Color online) pH sensor and connection.
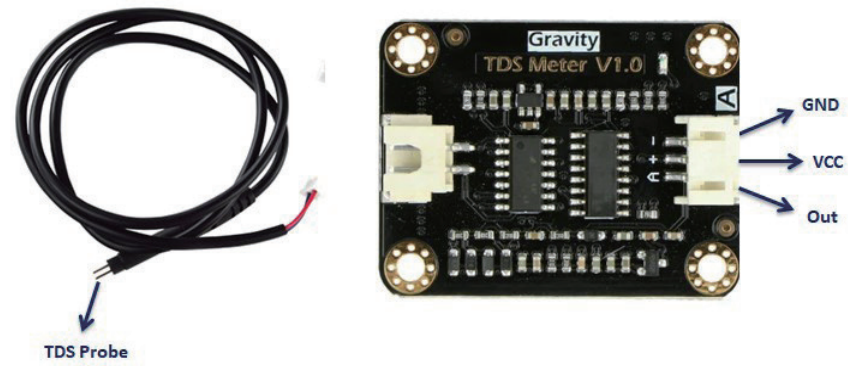

Fig. 6. (Color online) Typical TDS sensor board. 


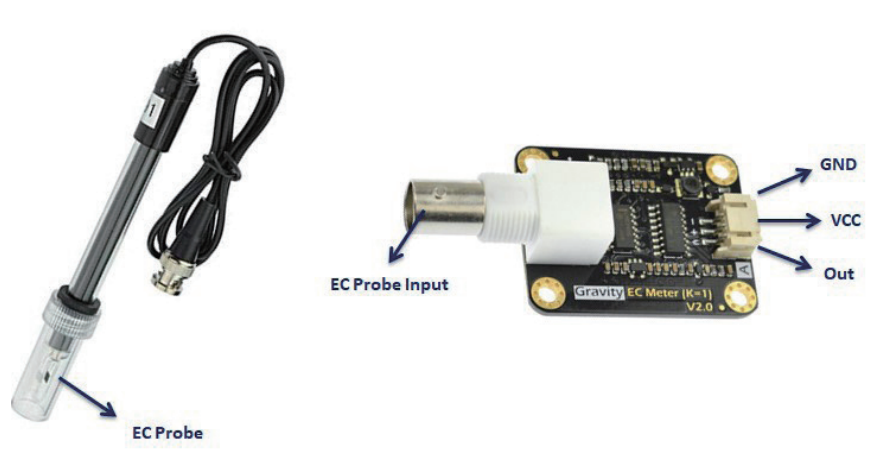

Fig. 7. (Color online) Common conductivity sensor.

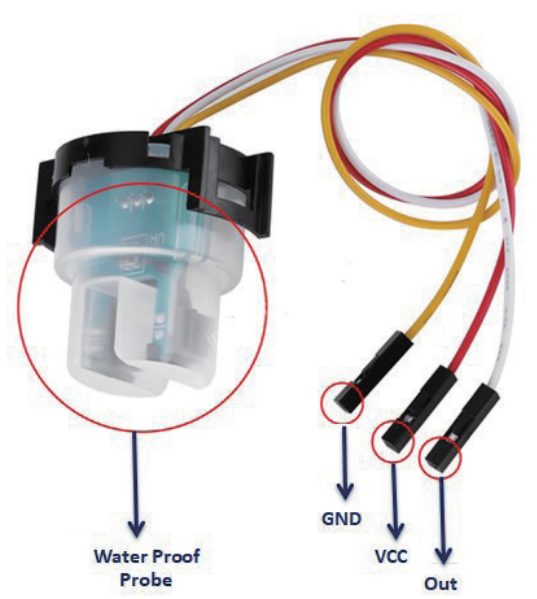

Fig. 8. (Color online) Typical turbidity sensor.

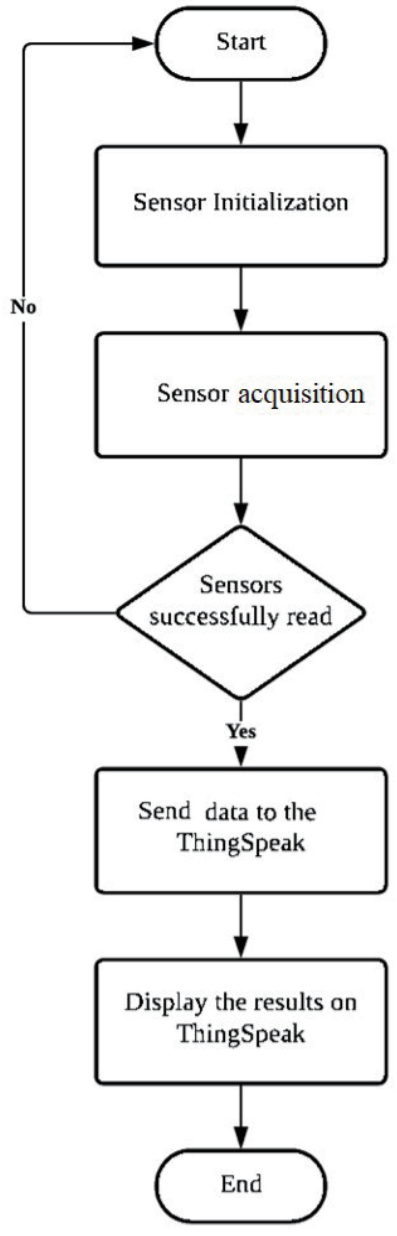

Fig. 9. (Color online) System flowchart.

\subsection{Software}

Figure 9 shows the process flow of the system used in this study. The process starts with sensor initialization, which is followed by data acquisition by the sensor. If the data acquisition is successful, the data will be sent to the ThingSpeak platform, which reads and records all the data acquired by the system. This platform also displays the data in the form of a table.

\section{Experiments}

In this study, we use two different types of water: tap water and filtered water. The tap water sample is obtained from the third floor of the Engineering Building of National Chin-Yi University of Technology. Both types of water sample are tested with the sensors described above. The preliminary stage shows that the quality of these two types of water is acceptable for humans to drink. The parameters in Table 1 are key determinants of water quality, and their acceptable ranges of values according to World Health Organization standards are also given in the table. 
Table 1

Common water quality parameters and their acceptable range of values.

\begin{tabular}{lc}
\hline Common water quality parameters & Acceptable ranges of value \\
\hline TDS & $<300-600 \mathrm{mg} / \mathrm{L}$ \\
Turbidity & $0-2.4 \mathrm{NTU}$ \\
$\mathrm{pH}$ & $6.5-8.5$ \\
Conductivity & $0-2500 \mu \mathrm{s} / \mathrm{cm}$ \\
Temperature & $20-25^{\circ} \mathrm{C}$ \\
\hline
\end{tabular}

\section{Results}

The water parameters are monitored and their values are plotted in real time. Each sensor is placed in both types of water at a temperature of $25{ }^{\circ} \mathrm{C}$. The temperature of the water has an important effect on the values of the other parameters.

\section{1 pH results}

Figures 10 and 11 show the $\mathrm{pH}$ results for the tap water and filtered water, respectively. As mentioned above, an acceptable $\mathrm{pH}$ for good water quality is around $6.5-8.5$ but both types of water have $\mathrm{pH}$ higher than 8.5 , meaning that the water is alkaline. Generally, humans should drink weakly alkaline water, so although the $\mathrm{pH}$ value of the experimental water here is slightly greater than 8.5, it is still suitable for people to drink. The sudden change seen in Fig. 10 was due to an error during the experiment.

\subsection{Turbidity results}

Figures 12 and 13 show the results of the turbidity of the tap water and filtered water, respectively. Turbidity is a measure of the transparency of the water, which remained constant

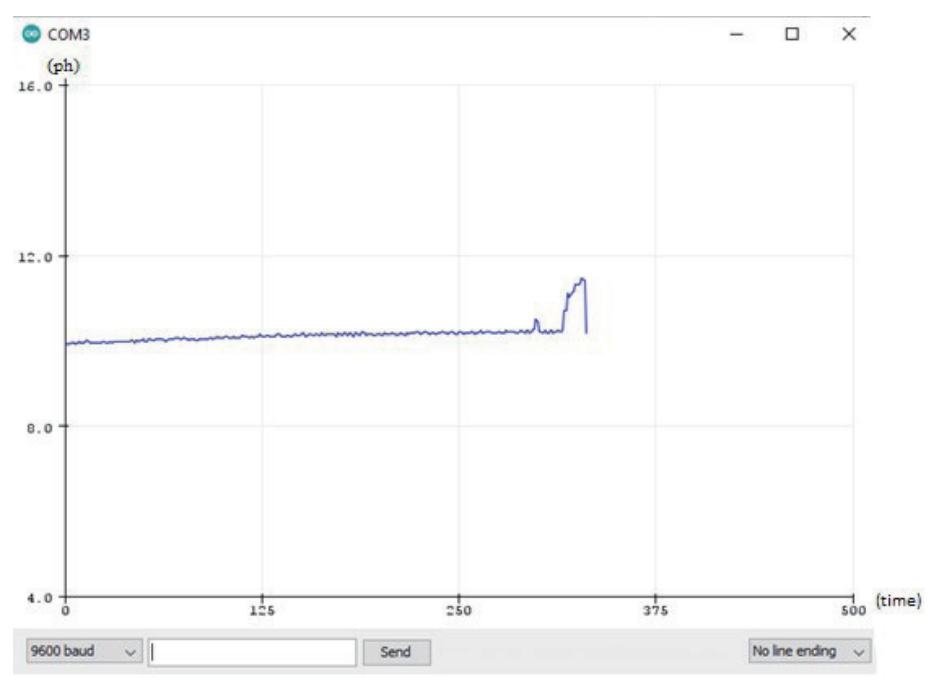

Fig. 10. (Color online) $\mathrm{pH}$ of tap water. 


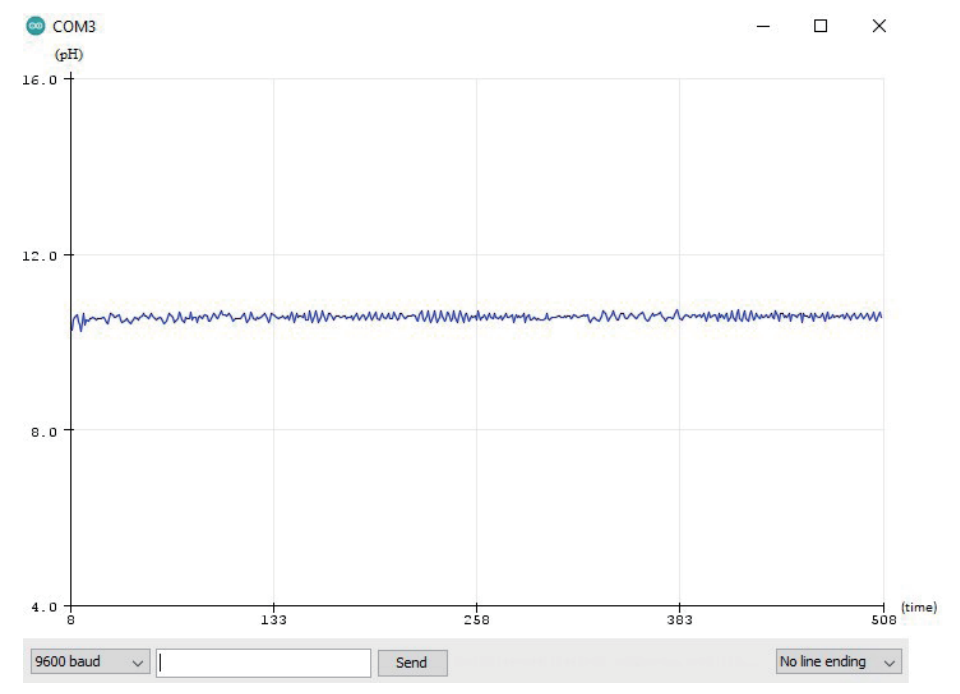

Fig. 11. (Color online) $\mathrm{pH}$ of filtered water.

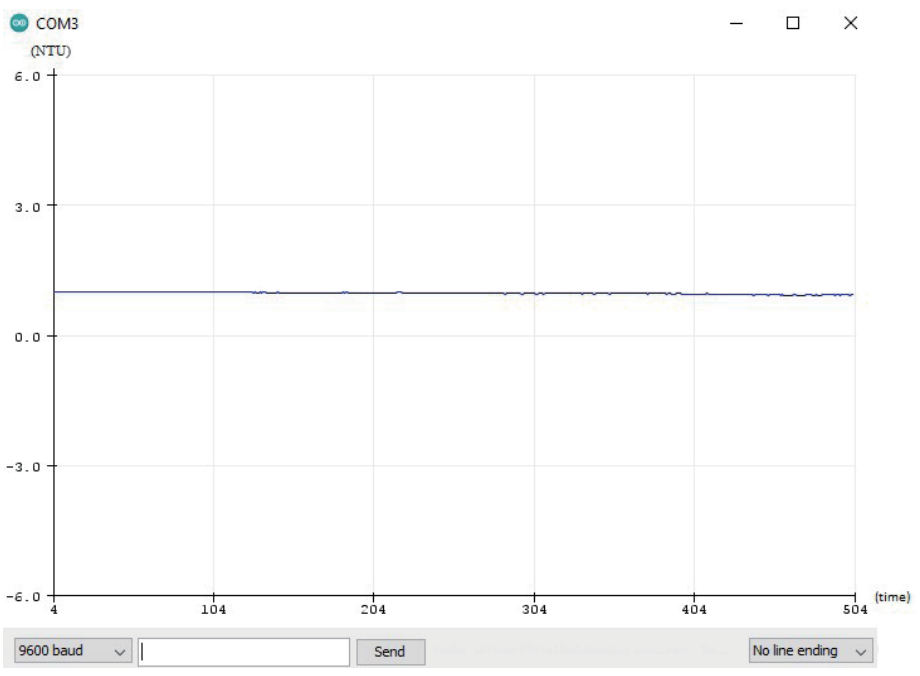

Fig. 12. (Color online) Turbidity of tap water.

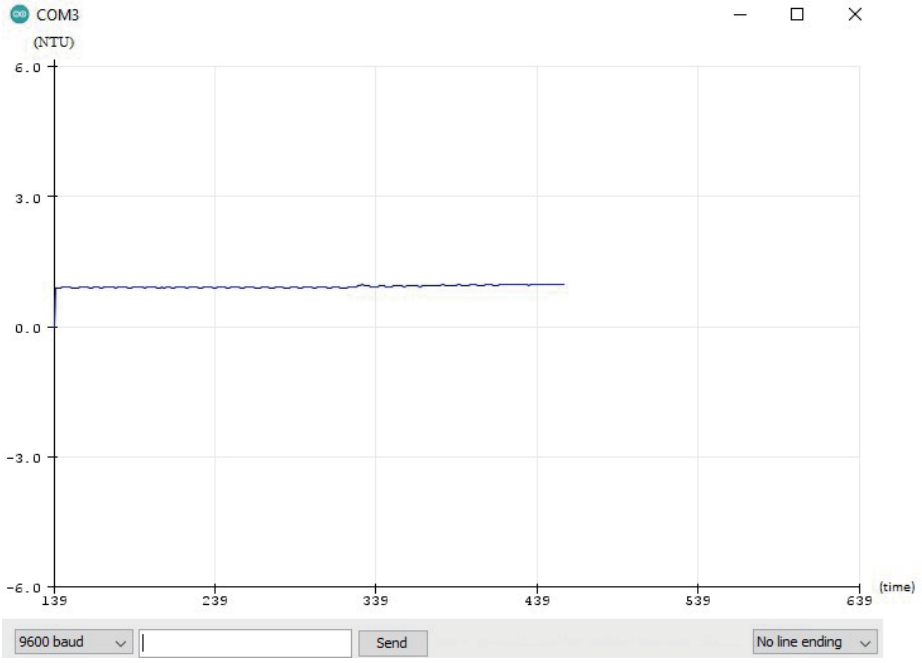

Fig. 13. (Color online) Turbidity of filtered water. 
while the sensor remained in the water samples. Both types of water sample are within the acceptable range for good water quality.

\subsection{Conductivity results}

Figures 14 and 15 show the results of conductivity for the tap water and filtered water, respectively. These results show that the electrical conductivity is in the range for good water quality. Conductivity is a numerical representation of the water's ability to transmit electricity. As mentioned above, the range of conductivity for good water quality is $0-2500 \mu \mathrm{s} / \mathrm{cm}$. Conductivity is influenced by the content of ions dissolved in the water. Thus, water with good quality is a poor conductor of electricity.

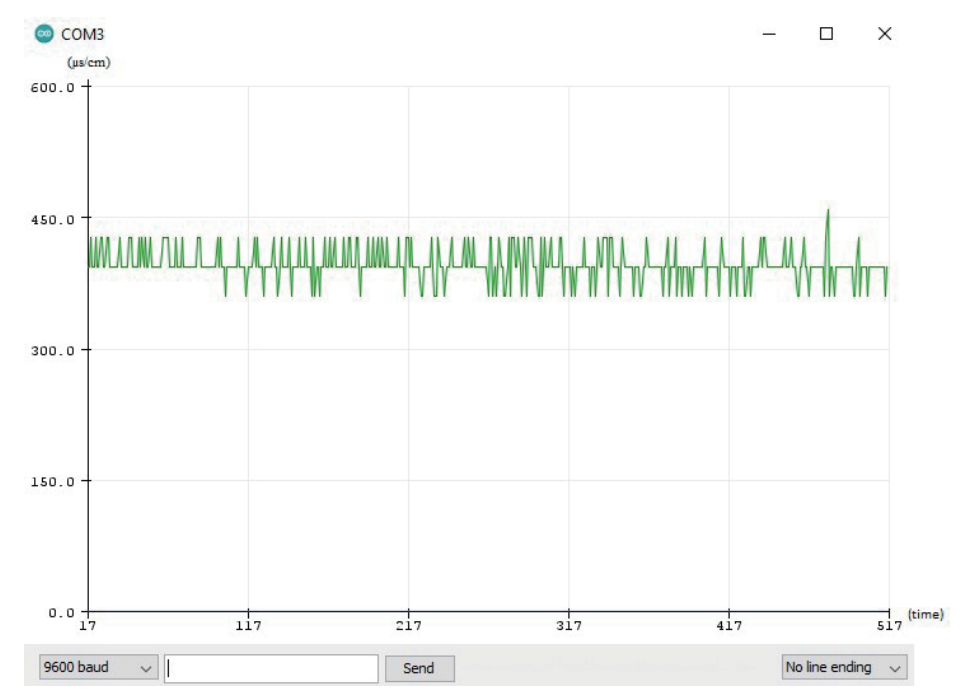

Fig. 14. (Color online) Conductivity of tap water.

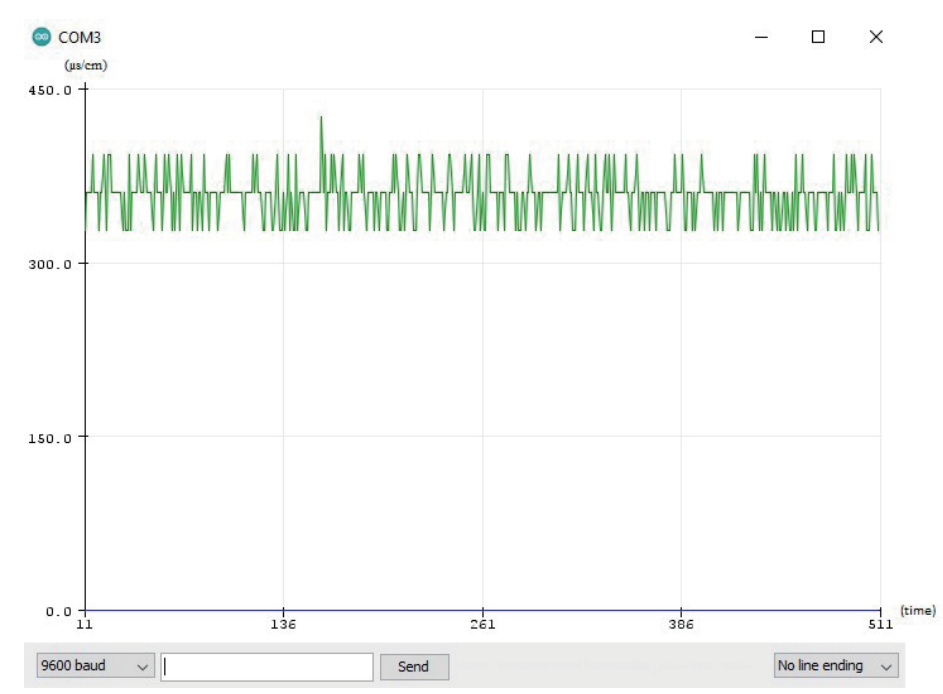

Fig. 15. (Color online) Conductivity of filtered water. 


\subsection{TDS results}

The TDS results for both types of water show that they are within the range of good water quality. The bump seen in the results is due to the probe being set to keep moving along with the depth of the water samples during the experiments. Figures 16 and 17 respectively show the TDS results for the tap water and filtered water.

\section{Water Quality Model for Running Water Based on Diversion Method}

After on-site surveys and multiparty discussions, we analyzed water intake methods. A water diversion method makes use of the flow induced by gravitational force. Water intake points include rivers and ditches. On the basis of our analysis, our team proposed the following water quality model for running water based on the diversion method.

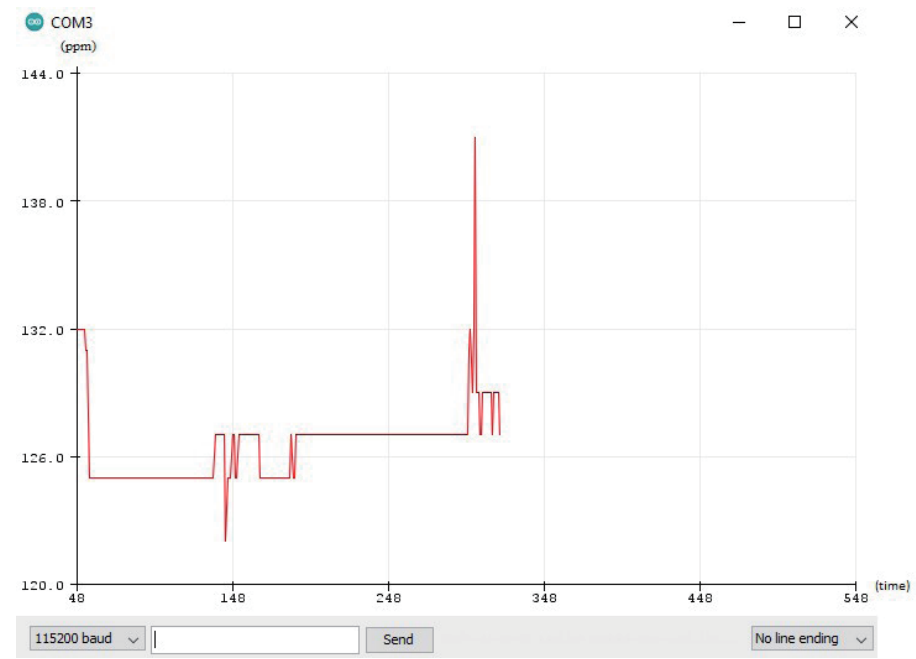

Fig. 16. (Color online) TDS result for tap water.

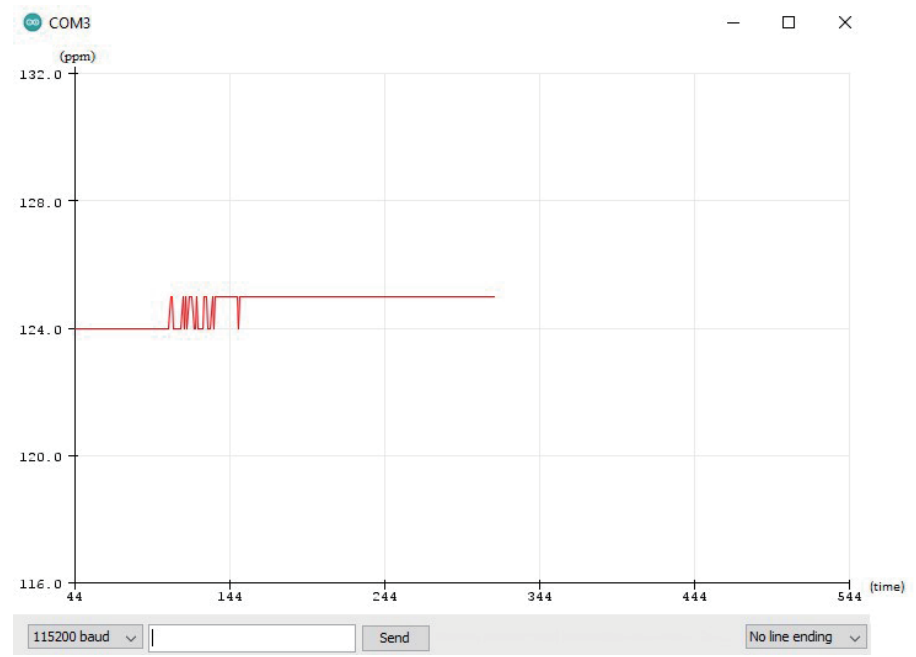

Fig. 17. (Color online) TDS result for filtered water. 


\subsection{Model principle}

The zero-dimensional water quality model is based on the principle of conservation of quality by mixing the inflowing pollutants uniformly throughout the entire water area, followed by the sedimentation and degradation of the pollutants by the water body. The concentration of pollutants in the water body after mixing is determined during the equilibrium period using the following formula.

$$
V d C=W d t-Q_{e} C d t-V C k d t
$$

$V$ : Water volume

$C$ : Concentration of water pollutants

$W$ : Pollutant load entering the water body during the period

$Q_{e}$ : Amount of water entering (outgoing) during the period

$k$ : Attenuation coefficient of total pollutants

After a certain period of time after being fully mixed, it can be assumed that the concentration of inflowing pollutants is stable and that the amounts of inflow and outflow water are the same. Then, the constant solution of the above formula is as follows.

$$
\bar{C}=\frac{\sum_{i=1}^{m} W_{i}}{\sum_{j=1}^{n} Q_{j}+k V}
$$

$\bar{C}:$ Average pollutant concentration in a certain period of time

$\sum_{i=1}^{m} W_{i}$ : Load of pollutants entering the river during the period

$\sum_{i=1}^{n} Q_{i}$ : Total amount of water entering (or outflowing) during the period

$k$ : Comprehensive attenuation coefficient of pollutants

$V$ : Total volume of water

The nonconstant solution of the model (for example, considering the daily changes in load and concentration) is

$$
C(t)=\frac{\sum_{i=1}^{m} W_{i}}{\sum_{i=1}^{n} Q_{i}+k V}\left\{1-\exp \left[-\left(\frac{\sum_{i=1}^{n} Q_{i}}{V}+k\right) t\right]\right\}+C_{0} \exp \left[-\left(\frac{\sum_{i=1}^{n} Q_{i}}{V}+k\right) t\right] .
$$

\subsection{Calibration of model parameters}

The annual total phosphorus content is $\sum_{i=1}^{m} W_{i}$ and the outflow is $\sum_{i=1}^{n} Q_{i}$ (the sum of the amount of sewage flowing into the river and the amount of through-flow) under the current 
working conditions (without diversion). Therefore, it can be considered that our proposed approach and various parameters can reflect and improve the regional water quality and can be used as an effective tool for calculation in research. The calculation period for determining the above model parameters is the whole year, and the calculation period is in days. Therefore, it is necessary to convert various parameters into daily average data. Rainfall is not yet considered in the forecast period, so rainfall runoff and runoff pollution are deducted from the outflow and pollution load.

\section{Conclusions}

We presented an IoT-based system for water quality monitoring that is cost-effective, efficient, and easily implemented in facilities. The sensors operated correctly and were able to detect the water quality of the two types of water source. This system also shows real-time data obtained from sensor readings. As shown by the results, both types of water were clear and had good quality; even the tap water was safe and clean.

When evaluating drinking water, the microbiological parameter is another parameter that indicates whether the water is safe to drink. This parameter is a measure of the number of bacteria contained in a water source. As future work, we hope to further develop our system to include this parameter. By our proposed method, the safety of drinking water can be ensured. Another task is to calculate the accuracy of the sensor readings.

\section{Acknowledgments}

We would like to thank Professor Wen-Tsai Sung for his expert advice and encouragement throughout this study. This research was supported by the Department of Electrical Engineering of National Chin-Yi University of Technology. The authors would also like to thank Takming University of Science and Technology, Taiwan, for supporting this research.

\section{References}

1 S. Sachio, A. Noertjahyana, and R. Lim: Proc. 2018 3rd Technology Innovation Management and Engineering Science Int. Conf. (2018) 1-5. https://doi.org/10.1109/TIMES-iCON.2018.8621630

2 N. Vijayakumar and R. Ramya: Proc.2015 Circuits, Power and Computing Technologies Int. Conf. (2015) 1-4. https://doi.org/10.1109/ICCPCT.2015.7159459

3 S. Jadhav, S. V. Patil, T. C. Thanuja, M. P. Shivu, and G. Shankar: Proc. 2018 Information, Communication, Engineering and Technology Int. Conf. (2018) 1-4. https://doi.org/10.1109/ICICET.2018.8533822

4 Y. Al-Khashab, R. Daoud, M. Majeed, and M. Yasen: Proc. 2019 Int. Conf. Computing and Information Science and Technology and Their Applications (2019) 1-5. https://doi.org/10.1109/ICCISTA.2019.8830662

5 F. Redwan, S. Rafid, A. H. Abrar, and B. B. Pathik: Proc. 2019 Int. Conf. Automation, Computational and Technology Management (2019) 137-142. https://doi.org/10.1109/ICACTM.2019.8776805

6 Y. K. Taru and A. Karwankar: Proc. 2017 Int. Conf. Computing Methodologies and Communication (2017) 416-419. https://doi.org/10.1109/ICCMC.2017.8282722

7 A. Mahmood, E. Sisinni, L. Guntupalli, R. Rondón, S. A. Hassan, and M. Gidlund: IEEE Trans. Ind. Inf. 15 (2019) 1425. https://doi.org/10.1109/TII.2018.2864681

8 A. Javed, H. Larijani, and A. Wixted: IT Professional 20 (2018) 30. https://doi.org/10.1109/MITP.2018.053891335

9 R. M. Sandoval, A. Garcia-Sanchez, J. Garcia-Haro, and T. M. Chen: IEEE Internet Things J. 5 (2018) 3114. https://doi.org/10.1109/JIOT.2018.2833289 
10 L. Feltrin, C. Buratti, E. Vinciarelli, R. De Bonis, and R. Verdone: LoRaWAN: IEEE Internet Things J. 5 (2018) 2249. https://doi.org/10.1109/JIOT.2018.2828867

11 J. Lim and Y. Han: IEEE Commun. Lett. 22 (2018) 800. https://doi.org/10.1109/LCOMM.2018.2797274

12 Y. Qian, L. Ma, and X. Liang: IEEE Commun. Lett. 22 (2018) 2230. https://doi.org/10.1109/ LCOMM.2018.2866820

13 H. Lee and K. Ke: IEEE Trans. Instrum. Meas. 67 (2018) 2177. https://doi.org/10.1109/TIM.2018.2814082

14 S. Popli, R. K. Jha, and S. Jain: IEEE Access 7 (2019) 16739. https://doi.org/10.1109/ACCESS.2018.2881533

\section{About the Authors}

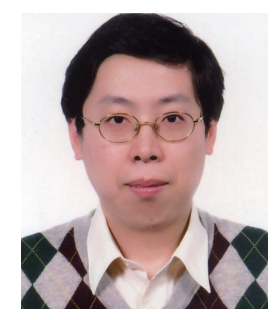

Wen-Tsai Sung is working in the Department of Electrical Engineering, National Chin-Yi University of Technology, as a distinguished professor and dean of research and development. He received his $\mathrm{PhD}$ and MS degrees from the Department of Electrical Engineering, National Central University, Taiwan, in 2007 and 2000, respectively. He won the 2009 JMBE Best Annual Excellent Paper Award and the Dragon Thesis Award sponsored by Acer Foundation. His research interests include artificial intelligence IoT (AIoT) and wireless sensor networks. (songchen@ncut.edu.tw)

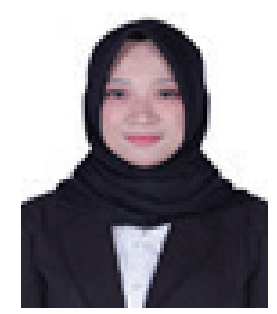

Fathria Nurul Fadillah received her BAS degree in telecommunication engineering from State Polytechnic of Sriwijaya, Indonesia, in 2019. She is currently studying for her master's degree in electrical engineering at National Chin-Yi University of Technology, Taiwan. Her research interests are in sensors, artificial intelligence, and IoT. (fathrianf@gmail.com)

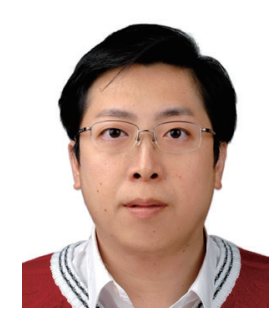

Sung-Jung Hsiao is working in the Department of Information Technology, Takming University of Science and Technology, as an assistant professor. He received his BS degree in electrical engineering from National Taipei University of Technology, Taiwan, in 1996, his MS degree in computer science and information engineering from National Central University, Taiwan, in 2003, and his $\mathrm{PhD}$ degree from the Department of Electrical Engineering, National Taipei University of Technology, in 2014. He has worked in research and design at Acer Universal Computer Co., Mitsubishi, and FIC. (sungjung@gs.takming.edu.tw) 